\begin{tabular}{l|l} 
Postprint Version & 1.0 \\
\hline Journal website & $\mathrm{http} / / /$ www.ingentaconnect.com \\
\hline Pubmed link & http://www.ncbi.nlm.nih.gov/entrez/query.fcgi?cmd=Retrieve\&db=pubmed\&do \\
pt=Abstract\&list uids=11330768\&query hl=61\&itool=pubmed docsum
\end{tabular}

Address for correspondence: J Dekker, Netherlands Institute of Primary Health Care, PO Box 1568, 3500 BN Utrecht, The Netherlands. e-mail: j.dekker@nivel.nl

\title{
The internal consistency and validity of the Self- assessment Parkinson's Disease Disability Scale
}

MAJE Biemans Practice for Physical Therapy and Manual Therapy 'de Traay' Driebergen,

J Dekker Netherlands Institute of Primary Health Care (NIVEL), Utrecht and Institute for Research in Extramural Medicine (EMGO-Institute), Vrije Universiteit Amsterdam

LHV van der Woude Institute for Fundamental and Clinical Human Movement Sciences, Faculty of Human Movement Sciences, Vrije Universiteit Amsterdam, The Netherlands

Objective: To test the consistency and validity of the Self-assessment Parkinson's Disease Disability Scale in patients with Parkinson's disease living at home.

Design: Patients with Parkinson's disease responded to a set of questionnaires. In addition, an observation of the performance of daily activities was carried out on a subgroup.

Setting and subjects: Patients with Parkinson's disease living at home $(n=142)$.

Measures: The Self-assessment Parkinson's Disease Disability Scale (SPDDS), the Hoehn \& Yahr Rating Scale (H\&Y), and the Sickness Impact Profile (SIP68). The observation concerned nine activities that correspond to items of the SPDDS questionnaire.

Results: Internal consistency of the SPDDS was very high (Cronbach's alpha $=0.97$; reliability rho $=0.97$ ). The items of the SPDDS are hierarchical (Loevinger's $H=0.64$ ): patients had least difficulty with 'washing' and 'brushing teeth' and most difficulty with 'turning in bed', 'travelling by public transport' and 'writing a letter'. Validity of the SPDDS was good: the relationship between the SPDDS questionnaire and the H\&Y rating scale, the SIP68 and the results of the observation was strong and significant.

Conclusion: The SPDDS is a unidimensional instrument measuring disabilities in Parkinson's disease patients living at home.

\section{INTRODUCTION}

In the long term, most patients with Parkinson's disease develop limitations in the performance of activities of daily living. The severity of disability increases with age and often makes it impossible for patients to live in their own home. Moreover, the inability to be independent in day-to- day living and social situations affects the patient's well-being in a negative way. ${ }^{1}$ For this reason, disabilities in activities of daily living should be carefully assessed.

Existing scales and questionnaires for the evaluation of patients with Parkinson's disease do not adequately address disabilities. The Unified Parkinson's Disease Rating Scale ${ }^{2}$ is a very popular instrument, but this scale does not differentiate between the impairments (such as tremor and rigidity) and disabilities (such as turning in bed). The scale contains just a few items that address disabilities. Another often-used instrument is the Hoehn \& Yahr Rating Scale. ${ }^{3}$ This scale provides a rating of the 
level of impairment (and not disability) due to the disease. The Northwestern University Disability Scale $^{4}$ is designed to address the disabilities. This is, however, a generic rather than a disease-specific scale. There are doubts about the reliability and responsiveness of this scale. ${ }^{5,6}$ Furthermore, a generic scale does not allow a detailed description of disabilities specifically occurring in Parkinson's disease (e.g. writing a letter). The Parkinson's Disease Questionnaire ${ }^{7}$ includes scales that measure quality of life. To a certain extent, this questionnaire addresses disabilities encountered during the performance of activities of daily living. The assessment of the disabilities, however, is confounded with the assessment of socio-emotional functioning.

The Self-assessment Parkinson's Disease Disabilities Scale (SPDDS) ${ }^{8,9}$ addresses a wide range of activities of daily living. Consequently, this scale seems to be suitable for a full evaluation of disabilities. The clinimetrical quality of this scale has, however, hardly been investigated. Brown et $a l^{8}$ claim that two subscales can be distinguished: 'gross' mobility and 'fine' co-ordination. The authors, however, do not present any data to support this claim. As regards the validity, Brown et al. ${ }^{10}$ report that the self-reported disabilities are largely similar to the disabilities reported by the patient's partner or by a neutral observer. No other studies on the validity of the SPDDS are available. Thus, although the SPDDS appears to be a suitable instrument for the measurement of disabilities in Parkinson's disease, its internal consistency and validity have hardly been studied.

The objective of the present study is (1) to establish whether the SPDDS questionnaire is a unidimensional or multidimensional instrument (internal consistency) and (2) to establish the validity of the SPDDS for the assessment of disabilities in patients with Parkinson's disease living at home.

\section{METHOD}

The SPDDS and other questionnaires were sent to 330 patients with Parkinson's disease. These were members of a patients' association. The internal consistency of the SPDDS was analysed with the aid of several statistics (see below). The validity of the SPDDS was studied by determining the relationship between the SPDDS and the Hoehn \& Yahr Rating Scale, and the Sickness Impact Profile.

In a subgroup $(n=30)$, the performance of a number of activities of daily living was observed and scored. These 30 patients were a stratified (according to the stages of Hoehn \& Yahr) random sample from the patients who participated in the questionnaire study. In this subgroup, the relationship between the observed performance and the self-reported performance (SPDDS) was determined; this provided a further test of the validity of the SPDDS.

The following measurement instruments were used:

-The $\operatorname{SPDDS}^{8,9}$ is a disease-specific questionnaire that consists of 24 items addressing activities of daily living (see Table 2). The patient indicates to what extent they are able to perform activities without help. The answers are rated on a five-point scale ranging from 'able to do alone without difficulty' to 'unable to do at all'. Originally, the SPDDS consisted of 25 items. One item has been added to the original list; this item addresses 'having a shower'. Two items ('getting into a bath' and 'getting out a bath') were dropped from the original list. These two items showed a high nonresponse: patients indicated that they did not own a bath.

- The Hoehn \& Yahr Rating Scale3 is used to stage the severity of Parkinson's disease. The stages range from I (mild symptoms on one side of the body) to V (full helplessness). Usually, health care providers score the H\&Y Rating Scale. In the present study, the patients assessed their own functioning. The approach was also used by Brown et al.10

- The SIP6811,12 is a shortened version of the Sickness Impact Profile. It includes 68 statements and is a generic measure of disability. The SIP68 overall score was used for comparison with the SPDDS.

-The performance (at home) of the following nine activities was observed: getting in and out of bed; getting up from and sitting down in an armchair; walking up and down the stairs; putting on and taking off one's coat; making a cup of tea or coffee; holding a cup and saucer; using the telephone; writing a letter; turning in bed. Occupational therapists $(n=4)$ made the observation. The observers were not aware of the Hoehn \& Yahr rating of the patients. The observers scored 
the performance of the activities on the same fivepoint scale as the SPDDS questionnaire. The observations took place $2-3$ months after completion of the questionnaire.

Internal consistency was statistically analysed as follows. Cronbach's alpha was computed. A value greater than 0.75 is generally considered sufficient. ${ }^{13}$ One of the characteristics of Cronbach's alpha is that it also has a high value in the event that the scale concerned includes a number of subgroups of mutually highly correlating items. Thus, a high value of Cronbach's alpha is not sufficient evidence for unidimensionality of a scale. ${ }^{13}$ For this reason, the Mokken Scale Analysis (MSA) ${ }^{14-16}$ was used as well. MSA is a nonparametric technique, based on the item-response theory. MSA generates a coefficient (Loevinger's $H$, scalability), indicating to what extent the items form a unidimensional hierarchy: when a patient indicates that he or she is able to carry out a particular activity, this patient provided the items show a perfect hierarchy - will also be able to perform activities that are 'easier'. The minimum required value for Loevinger's $H$ is $0.50 .{ }^{15}$ In the event that the items form a unidimensional hierarchy, the internal consistency of the scale can be computed. Internal consistency within the MSA is computed using the reliability rho, which is comparable with Cronbach's alpha. The required minimum is $0.75 .{ }^{15}$ The statistical analysis of the validity of the SPDDS was as follows. The relationship between the SPDDS and the SIP68 was tested by means of the Spearman correlation coefficient. A coefficient of 0.70 or higher indicates a high association. ${ }^{17}$

The relationship between the Hoehn \& Yahr Rating Scale and the SPDDS was determined using analysis of variance. The relationship (on item level) between observation and self-report (SPDDS) was tested using the intraclass correlation coefficient (ICC 3,1) ${ }^{18} \mathrm{~A}$ value of 0.80 or higher indicates a high association. ${ }^{19}$

The Mokken Scale Analysis was performed using the programme MSP version 3.0. ${ }^{15}$ All other analyses were performed using SPSS version 7.5 for Windows.

\section{RESULTS}

\section{Patients}

The questionnaires were sent to 330 patients, 192 questionnaires were returned and 142 questionnaires (43\%) were fully completed (i.e. no single item of the SPDDS missing). The analysis was performed on the data provided by these 142 patients. There was no significant difference in the H\&Y scores in these 142 patients and the 50 patients that were excluded from the analysis $\left(\mathrm{chi}^{2}=5 ; p\right.$ $=0.8$ ). Of these 142 patients, $57 \%$ were male and $43 \%$ female (Table 1). Ages ranged from 40 to 85: $36 \%$ were younger than 65 and $64 \%$ were older than 65 . Fifteen per cent of the patients were living alone, the other $85 \%$ were living with a partner or someone else. Most patients were in stage I, II or III of the H\&Y scale.

\section{Internal consistency}

Cronbach's alpha coefficient was 0.97. Internal consistency of the SPDDS was confirmed by the Mokken analysis: Loevinger's $H$ was 0.64 and reliability rho was 0.97 . These results imply that the items of the SPDDS form a unidimensional hierarchy.

Table 2 shows the mean, the standard deviation and the scalability for each item of the SPDDS. The items are arranged according to degree of difficulty (from least disability to most disability). Table 2 shows that the scalability of each individual item (i.e. the degree to which an item fits into the unidimensional hierarchy) is above the minimum value of 0.50 . Patients had the least problems with 'washing' and 'brushing their teeth'. Activities such as 'getting dressed and undressed' and 'getting out of bed' showed an average degree of difficulty. The items 'turning in bed', 'travelling by public transport' and 'writing letters' were the most difficult activities.

\section{Validity}

Figure 1 shows the mean and the standard deviation of the SPDDS questionnaire, itemized for the H\&Y stages. The SPDDS score increased by stage according to H\&Y $(F=104.85, \mathrm{df}=141, p<$ 0.0001). In stage I, the mean SPDDS score was 30.3 (SD 5.8), in stage II 36.9 (SD 7.4), in stage III 49.3 (SD 10.3), in stage IV 72.3 (SD 15.6) and in stage V 95.2 (SD 19.6). The overall mean score was 48.7 (SD 21.3). 
The Spearman correlation between the SPDDS questionnaire and the score on the SIP68 was determined; the correlation coefficient was $0.83(p<0.01)$. This indicates a high association. This is illustrated in a scatter plot (Figure 2).

In a subgroup $(n=30)$ a comparison on the item level was made between the reported score and the score obtained during observation (Table 3). The number of subjects per item compared ranged from 18 to 29 . The intraclass correlation coefficient varied from 0.52 to 0.82 . The ICC values showed a moderate to high positive association.

\section{DISCUSSION}

The internal consistency of the SPDDS questionnaire was very high. Brown et al. ${ }^{8}$ claimed the existence of two subscales, namely 'fine' co-ordination and 'gross' mobility. This distinction was not found in the present study. Both Cronbach's alpha and the Mokken Scale Analysis indicated that the SPDDS is a unidimensional scale, without subscales.

The high internal consistency may be interpreted as a sign of redundancy of items. We have conducted several analyses, omitting items from the SPDDS. In these analyses, the internal consistency of the scale remained high (with eight items internal consistency was still $>0.80$, not reported in the Results section). Thus, one could omit items from the SPDDS. However, we chose not to reduce the number of items, because we wanted to acquire a comprehensive, overall picture of the disabilities of Parkinson patients, which requires addressing a range of items. This approach has distinct advantages. For example, our data show (see Table 2) that the patients had the least difficulty in the performance of 'washing face and hands', 'brushing your teeth' and 'using a telephone'. The items 'getting undressed', 'holding and reading a newspaper' and 'holding a cup and saucer' were of intermediate difficulty, while most difficulty was encountered in the performance of 'turning over in bed', 'travelling by public transport' and 'writing a letter'. Thus, considerable differentiation exists in the difficulty of various activities: this differentiation can only be discovered using a wide range of activities and thus items.

The skewed distribution of the patients over the H\&Y stages did not affect the internal consistency. We have repeated our analysis in a subsample of 45 patients, with nine patients in each stage according to H\&Y. In this analysis, Cronbach's alpha and the reliability rho remained high (both 0.98 ; not reported in the Results section).

The validity of the SPDDS - as determined by the association with other assessment instruments was shown to be good. There was a strong relationship between the SPDDS questionnaire and the stages of the diseases according to H\&Y. The average SPDDS scores show a clear increase with the H\&Y stages. The SIP68 - which repeatedly has been shown a valid instrument ${ }^{11,12}$ - showed a high correlation with the SPDDS. At the level of individual items, the SPDDS scores do correspond well with the results of the observations. It is an evident weakness of the present study that the observations were performed 2-3 months after completion of the SPDDS. Nevertheless, the self-reported and the observed scores corresponded well. To the extent that the scores were not equal, the observation generally indicated more disability than the self-report (not shown in the Results section). The reason for this could be that the patients completed the survey when they were 'feeling well' and perceived their situation more positively than it generally will be. Asking the respondents to complete the survey at a fixed day, thus prohibiting them to select a 'good' day, can solve this problem.

The present study was conducted on patients with Parkinson's disease, living in their own home. The questionnaires were sent to 330 patients, 192 of whom responded. It is possible that the most seriously ill patients did not manage to complete and send the survey. Thus, our study could give a too positive picture of disability in Parkinson patients living at home. A further 50 patients were excluded from the analysis because they did not complete all items of the SPDDS. The H\&Y scores of these patients did not differ significally from the remaining 142 patients ( $p<0.8$; see Results section). Furthermore, the usable response of $142(43 \%)$ is comparable to another survey among Parkinson's patients. ${ }^{20}$ The average age of the patients that participated in this survey was 66; this is reasonably consistent with Jenkinson et al. ${ }^{21}$ and Montgomery et al. ${ }^{22}$ who found an average age of 70.3 and 61.5 years, respectively. Most respondents were in stage I, II or III of the H\&Y Rating Scale. This distribution is consistent with the distribution found in Brown et al. ${ }^{10}$ Therefore, we do not believe that the present study is seriously biased by patient selection. 
The present study used a cross-sectional design. Thus we were not able to address the issue of responsiveness. Responsiveness refers to the ability of an instrument to pick up clinically meaningful changes in the status of the patient. Evaluation of responsiveness requires a longitudinal design. It is recommended that the responsiveness of the SPDDS be evaluated in future, longitudinal research.

We conclude that the SPDDS is an internally consistent and valid instrument for the assessment of the disease-specific disabilities in patients with Parkinson's disease living at home.

\section{REFERENCES}

1 Emanuels-Zuurveen ES, Brouwer WH, Lakke JPWF, Bouhuys AL. Lichamelijke symptomen van de ziekte van Parkinson en de score op de Beck's Depression inventory. T Gerontol Geriatr 1991; 22: 134-38.

2 Martinez-martin P, Gil-Nagel A, Morlan Gracia L, Balseiro Gomez J, Martinez-Sarries J, Bermejo F and the Cooperative Multicentric Group. Unified Parkinson's Disease Rating Scale characteristics and structure. Mov Disord 1994; 1: 76-83.

3 Hoehn MM, Yahr MD. Parkinsonism: onset, progression and mortality. Neurology 1967; 17: 427-42.

4 Canter CJ, de la Torre R, Mier M. A method of evaluating disability in patients with Parkinson's disease. J Nerv Mental Disord 1961; 133: 143-47.

5 Marsden C.D., Schachter M. Assessment of extrapyramidal disorders. Br J Clin Pharmacol 1981; 11: $129-51$.

6 Diamond SG, Markham CH. Evaluating the evaluations: or how to weigh the scales of Parkinsonian disability. Neurology 1983; 33: 1089-92.

7 Peto V, Jenkinson C, Fitzpatrick R, Greenhall R.

The development and validation of a short measure of functioning and well being for individuals with Parkinson's disease. Qual Life Res 1995; 4: 241-48.

8 Brown RG, Maccarthy B, Gotham AM, Der GJ, Marsden CD. Depression and disability in Parkinson's disease: a follow-up of 132 cases. Psychol Med 1988; 18: 49-55.

9 Wade DT. Measurement in neurological rehabilitation. Oxford: Oxford University Press, 1992.

10 Brown RG, MacCarthy B, Jahanshahi M, Marsden CD. Accuracy of self-reported disability in patients with Parkinsonism. Arch Neurol 1989; 46: 955-9.

11 Bruin AF de, Buys M, Witte LP de, Diederiks JPM. The Sickness Impact Profile: SIP68, a short generic version. First evaluation of the reliability and reproducibility. J Clin Epidemiol 1994; 8: 86371.

12 Marcel WM, Post MWM, Bruin A de, Witte L de, Schrijvers G. The SIP68: a measure of healthrelated functional status in rehabilitation medicine. Arch Phys Med Rehabil 1996; 77: 440-45.

13 Streiner DL, Norman GR. Health measurement scales: a practical guide to their development and use, second edition. Oxford: Oxford University Press, 1995.

14 Mokken, RJ. A theory and procedure of scale analyses. The Hague: Mouton, 1971.

15 Molenaar IW, Debets P, Sijtsma K, Hemker BT. User's manual MSP: a program for Mokken Scale Analysis for polytomous items, version 3.0. Groningen: ProGamma, 1994.

16 Hemker BT, Sijtsma K, Molenaar IW. Selection of unidimensional scales from a multidimensional item bank in the polytomous Mokken IRT Model. Appl Psychol Measurement 1995; 337-52.

17 Hinkel DE, Wiersma W, Jurs SG. Applied statistics for the behavioral sciences, third edition. Boston: Houghton Mifflin, 1994.

18 Rankin G, Stokes M. Reliability of assessment tools in rehabilitation: an illustration of appropriate statistical analyses. Clin Rehabil 1998; 12: 187-99.

19 Landis RJ, Koch GG. The measurement of observer agreement for categorical data. Biometrics 1977; 33: 159-74.

20 Wimmers RH, Kansma YPT. Een enquête naar handelingsproblemen bij patiënten met de ziekte van Parkinson. Ned Tijdschrift Fysiother 1998; 3: 54-61.

21 Jenkinson C, Fitzpatrick R, Peto V, Greenhall R, Hyman N. The Parkinson's Disease Questionnaire (PDG-39): development and validation of a Parkinson's disease summary index score. Age Ageing 1997; 26: 353-57.

22 Montgomery GK, Norman C, Reynolds MD. Compliance, reliability, and validity of selfmonitoring for physical disturbances of Parkinson's disease. J Nerv Ment Disord 1990; 178: 636-41. 